\title{
RESistênCIA CRUZAda da losNa-BRANCA (Parthenium hysterophorus) aOS Herbicidas INIBIDORES da ENZIMA ACETOlactato Sintase ${ }^{1}$
}

\author{
Ragweed Parthenium (Parthenium hysterophorus) Cross-resistance to Acetolactate Synthase \\ Inhibiting Herbicides
}

GAZZIERO, D.L.P.․․ BRIGHENTI, A.M. ${ }^{2}$ e VOLL, E. ${ }^{2}$

\begin{abstract}
RESUMO - A aplicação de um mesmo herbicida, ou de herbicidas com o mesmo mecanismo de ação, durante anos consecutivos, numa mesma área, pode resultar na seleção de biótipos de plantas daninhas resistentes a herbicidas. O objetivo deste trabalho foi confirmar a resistência de um biótipo da planta daninha losna-branca (Parthenium hysterophorus) aos herbicidas inibidores da enzima acetolactato sintase (ALS), proveniente de uma propriedade rural no município de Mandaguari, norte do Estado do Paraná. Plantas com suspeita de resistência foram tratadas com diversos herbicidas e doses e comparadas com plantas de uma população suscetível. Os tratamentos foram as doses recomendadas dos herbicidas, duas e quatro vezes superiores à dose recomendada. Os produtos e as doses aplicadas foram cloransulammethyl a 0,0; 33,6; 67,2; e 134,4 g i.a. ha-1 mais o adjuvante Agral a 0,2\% v/v, chlorimuronethyl a 0,0; 20,0; 40,0; e 80,0 g i.a. ha-1, imazethapyr a 0,0; 100,0; 200,0; e 400,0 g i.a. ha-1 e iodosulfuron-methyl-sodium mais foramsulfuron a 0,0; 3,0 +45,0 g i.a. ha-1 (150,0 g p.c. ha-1); $6,0+90,0$ g i.a. ha-1 $(300,0$ g p.c. ha-1); e $12,0+180,0$ g i.a. ha-1 $(600,0$ g p.c. ha-1). Foi acrescentado um tratamento com o herbicida 2,4-D na dose de 536,0 g e.a. ha ${ }^{-1}$. As curvas de dose-resposta do biótipo resistente foram inferiores às do biótipo suscetivel em todas as doses e herbicidas estudados. O biótipo de losna-branca foi confirmado como resistente aos herbicidas inibidores da ALS. A ocorrência de resistência cruzada foi observada em relação aos herbicidas pertencentes aos grupos químicos das imidazolinonas (imazethapyr), triazolopirimidinas (cloransulam-methyl) e sulfoniluréias (chlorimuron-ethyl e iodosulfuron-methyl-sodium mais foramsulfuron). O herbicida 2,4-D, apresentou alto índice de controle de ambos os biótipos de losna-branca avaliados, confirmando que esse mecanismo de ação do herbicida é uma importante alternativa para manejar áreas com problemas de resistência.
\end{abstract}

Palavras-chave: imidazolinonas, sulfoniluréias, triazolopirimidinas, reguladores de crescimento, curvas de doseresposta.

\begin{abstract}
Weed control using herbicide application is a common agricultural practice. However, the application of the same herbicide or herbicides with the same mechanism of action, for consecutive years, in the same area, can result in the selection of herbicide resistant biotypes. The aim of this work was to confirm the resistance of a ragweed (Parthenium hysterophorus) biotype to acetolactate synthase (ALS) inhibiting herbicides. The plants were collected on a farm in Mandaguari, north of Parana State, Brazil. Plants with suspicious resistance were treated with several herbicides and rates and compared with those of a susceptible population. The herbicide treatments were established considering the recommended rates, double and four times higher than the recommended rate as follows: cloransulam-methyl 0.0, 33.6, 67.2 and $134.4 \mathrm{~g}$ a.i. $h \mathrm{a}^{-1}$ plus adjuvant $0.2 \% \mathrm{v} / \mathrm{v}$, chlorimuron-ethyl $0.0,20.0,40.0$ and $80.0 \mathrm{~g}$ a.i., imazethapyr 0.0, $100.0,200.0$ and $400.0 \mathrm{~g}$ a.i. ha $\mathrm{h}^{-1}$, iodosulfuron-methyl-sodium plus foramsulfuron $0.0,3.0+45.0$ ga.i. ha- $\left(150.0 \mathrm{~g}\right.$ c.p. $\left.h a^{-1}\right), 6.0+90.0 \mathrm{~g}$ a.i. $\mathrm{ha}^{-1}\left(300.0 \mathrm{~g} \mathrm{c.p.} \mathrm{ha^{-1 } )}\right.$ and $12.0+180.0 \mathrm{~g}$ a.i. ha $\mathrm{h}^{1}\left(600.0 \mathrm{~g}\right.$ c.p. $\left.\mathrm{ha}^{-1}\right)$. In addition, a treatment with 2,4-D (536.0 g a.e. $\left.\mathrm{ha}^{1}\right)$ was applied. Resistant plant dose-response curves presented lower values when compared to the susceptible population, in all rates and herbicides studied. The ragweed biotype was confirmed as resistant to the ALS inhibiting herbicides. Cross-resistance was observed with
\end{abstract}

1 Recebido para publicação em 12.7.2005 e na forma revisada em 24.2.2006.

Eng.-Agr., Doutor, Pesquisador da Embrapa Soja, Caixa Postal 231,86001-970 Londrina-PR, <gazziero@ cnpso.embrapa.br>. 
herbicides belonging to the chemical groups of imidazolinones (imazethapyr), triazolopyrimidines (cloransulam-methyl), sulfonylureas (chlorimuron-ethyl and iodosulfuron-methyl-sodium plus foramsulfuron). 2,4-D has a different mechanism of action, presenting high values of control, and thus being a management alternative in areas with ragweed resistant population.

Keywords: imidazolinones, sulfonylureas, triazolopyrimidines, growth regulators, dose-response curves.

\section{INTRODUÇÃO}

As plantas resistentes surgem, principalmente, em áreas de monocultura onde as aplicações repetidas de um único e efetivo herbicida, ou de herbicidas de mesmo mecanismo de ação se estendem por vários anos, causando mudanças na flora infestante (Maxwell et al., 1990; Blair, 1991). Certos indivíduos (biótipos), dentro da população, são capazes de sobreviver a aplicações desses herbicidas mais do que outros.

No Brasil, o primeiro caso confirmado de uma população de planta daninha resistente a herbicidas foi relatado por Christoffoleti et al. (1996), com a planta daninha picão-preto (Bidens pilosa), em regiões produtoras de soja.

Gazziero et al. (1998) e Vidal \& Merotto Jr. (1999) relataram populações de Euphorbia heterophylla resistentes a herbicidas inibidores da ALS em algumas regiões produtoras de soja no Brasil. Monqueiro et al. (2000) também confirmaram a existência de populações resistentes de Bidens pilosa e Bidens subalternans em lavouras de soja no Mato Grosso do Sul.

Em São Gabriel D’Oeste, MS, foi confirmada a resistência de Bidens subalternans aos herbicidas inibidores da ALS, pertencentes aos grupos químicos imidazolinonas e sulfoniluréias (Gelmini et al., 2002).

Gazziero et al. (2000) confirmaram a resistência de um biótipo da planta daninha capim-marmelada (Brachiaria plantaginea) aos herbicidas inibidores da ACCase provenientes de uma área de cultura de soja da região sudoeste do Estado do Paraná.

Depois disso, outros trabalhos confirmaram a resistência de plantas daninhas como o capim-arroz (Merotto Jr. et al., 2000; Eberhardt et al., 2000), o capim-colchão (Buzatti et al., 2002), a sagitária (Noldin et al., 2000), o junquinho (Noldin et al., 2002a), o cuminho (Noldin et al., 2002b) e o azevém (Roman et al., 2004; Vargas et al., 2004).

Em relação à losna-branca (Parthenium hysterophorus), foram observadas áreas de escape dessa planta daninha na região norte do Estado do Paraná, devido a falhas de controle químico em áreas de produção comercial, com aumento considerável da sua população. Essa espécie pertence à família Asteraceae, sendo nativa do Continente Americano (Kissmann \& Groth, 1999). Sua reprodução se dá por sementes, vegetando quase o ano inteiro e com formação de densas infestações com longo florescimento. Foi introduzida acidentalmente em certos países da Ásia e da Oceania, tendo se tornado uma séria infestante na região de Queensland (Austrália) e na Índia. No Brasil, a população de losna-branca tem crescido, principalmente, no norte do Estado do Paraná, em São Paulo e Mato Grosso do Sul. Em Corumbá predominam plantas com flores amarelas e, nos Estados do Paraná e São Paulo, com flores brancas. As plantas contêm uma substância chamada parthenina, que age como sedativo no sistema nervoso e, assim, tem sido usada na medicina popular. Seus tricomas contêm lactonas alergógenas, que produzem dermatites ao contato, em pessoas sensíveis.

Njoroge (1991) afirmou ter encontrado plantas de losna-branca tolerantes ao paraquat em lavouras de café, no Quênia. Por sua vez, Adkins et al. (1997) não verificaram resultado positivo ao estudar a resistência de duas coleções de losna-branca à atrazina, provenientes de áreas produtoras de grãos na Austrália.

O presente trabalho objetivou analisar plantas provenientes de áreas onde ocorreram falhas de controle e determinar a possibilidade da manifestação da resistência da losnabranca aos herbicidas inibidores da enzima acetolactato sintase. 


\section{MATERIAL E MÉTODOS}

Sementes de losna-branca foram colhidas em uma área de produção de soja cujo histórico indicava problemas de controle com o uso continuado de produtos inibidores da ALS. Sementes de um biótipo supostamente suscetível foram coletadas de um local não cultivado. O experimento foi conduzido em casa de vegetação, utilizando plantas de losna branca provenientes de uma propriedade rural do município de Mandaguari, norte do Estado do Paraná.

Os dois biótipos foram semeados separadamente em 2 agosto de 2004, em vasos plásticos com capacidade de $3 \mathrm{~kg}$ de terra, preparada com $20 \%$ de matéria orgânica (esterco curtido), 25\% de areia lavada e 55\% de solo comum, previamente esterilizado com brometo de metila. Regularmente era feita a irrigação, para que a umidade do solo se mantivesse próxima à capacidade de campo. Após a germinação, foi realizado o desbaste, mantendo-se duas plantas por vaso. A aplicação dos herbicidas foi realizada em $30 \mathrm{de}$ agosto, correspondendo a 24 dias da emergência, quando as plantas estavam com quatro a seis folhas. O delineamento experimental foi o de blocos ao acaso, com quatro repetições. Os tratamentos foram estabelecidos considerando a dose recomendada e duas e quatro vezes esta dose. Os herbicidas e as doses aplicadas foram cloransulam-methyl a 0,$0 ; 33,6 ; 67,2 ;$ e 134,4 g i.a. ha ${ }^{-1}$ mais o adjuvante Agral 0,2\% v/v, chlorimuron-ethyl a 0,$0 ; 20,0 ; 40,0$; e 80,0 gi.a. ha ${ }^{-1}$, imazethapyr a 0,$0 ; 100,0 ; 200,0 ;$ e 400,0 g i.a. ha-1 e iodosulfuron-methyl-sodium + foramsulfuron a 0,$0 ; 3,0+45,0$ g i.a. ha ${ }^{-1}$ (150 g p.c. ha-1); $6,0+90,0$ g i.a. ha-1 $(300,0$ g p.c. ha-1); e 12,0 $+180,0$ g i.a. ha-1 $\left(600,0\right.$ g p.c. ha $\left.{ }^{-1}\right)$. Além disso, foi acrescentado um tratamento com o herbicida 2,4-D na dose de 536,0 g e.a. ha-1. $\mathrm{Na}$ aplicação dos herbicidas foi utilizado pulverizador costal a $\mathrm{CO}_{2}$, com bico 110.02 , a pressão de $207 \mathrm{kPa}$ e volume de calda de $160 \mathrm{~L} \mathrm{ha}^{-1}$.

Avaliações visuais de controle foram feitas aos 7, 14 e 21 dias após a aplicação (DAT), utilizando a escala percentual, sendo obtida a fitomassa seca aos 30 dias após a aplicação dos tratamentos.
O diferencial de controle (S-R) foi calculado subtraindo-se o valor do percentual de controle do biótipo suscetível do percentual de controle do biótipo resistente.

Os valores de $\mathrm{GR}_{50}$, ou seja, a dose necessária para propiciar 50\% da redução da fitomassa seca do biótipo resistente e do biótipo suscetivel, foram obtidos a partir dos modelos ajustados; as relações médias de $\mathrm{GR}_{50}$ foram calculadas para cada produto, dividindo-se o $\mathrm{GR}_{50}$ do biótipo resistente pelo do biótipo suscetivel (R/S).

Foram ajustados modelos de regressão aos dados (raiz quadrada $\left(\mathrm{Y}=\mathrm{a}+\mathrm{bX}^{1 / 2}+\mathrm{cX}\right)$ ) observados, tendo como variável a resposta da fitomassa seca.

\section{RESULTADOS E DISCUSSÃO}

Na Tabela 1 são mostrados os valores de porcentagem de controle da losna-branca aos 7, 14 e 21 DAT. Todos os herbicidas aplicados na dose recomendada proporcionaram controle das plantas suscetiveis acima de $86 \%$, aos 14 DAT. Para essa mesma dose, os percentuais atingidos foram acima de 98\% aos 21 DAT, exceto para o imazethapyr (86\%). Para as doses duas e quatro vezes a recomendada, o controle foi acima de 90\%, aos 14 DAT. Com relação às plantas com suspeita de resistência, não houve qualquer sintoma de injúria relativa à aplicação dos herbicidas, sendo igual a zero o percentual de controle obtido. O herbicida com maior diferencial de controle aos 21 DAT foi o cloransulam-methyl (99\%), seguido de chlorimuron-ethyl (98,6\%), iodosulfuronmethyl-sodium mais foramsulfuron (98\%) e imazethapyr $(86,6 \%)$ (Tabela 2). Brighenti et al. (2004) avaliaram o controle da losnabranca por meio da aplicação de diversos herbicidas e verificaram que, dos produtos avaliados, o cloransulam-methyl (39 g i.a. ha ${ }^{-1}$ ) e o 2,4-D (1.005 g e.a. ha ${ }^{-1}$ ) foram os mais eficazes no controle de populações suscetiveis dessa planta daninha.

$\mathrm{O}$ valor de $\mathrm{GR}_{50}$ relativo ao herbicida cloransulam-methyl foi superior à maior dose aplicada (134,4 g i.a. ha h $\left.^{-1}\right)$ para as plantas com suspeita de resistência e igual a 7,13 g i.a. ha-1 para as suscetiveis (Tabela 3 ). 
Tabela 1 - Porcentagem de controle da losna-branca resistente (R) e suscetível (S) aos 7, 14 e 21 dias após a aplicação dos tratamentos (DAT), em função da dose zero (0), dose recomendada (1X), duas vezes a dose recomendada (2X) e quatro vezes a dose recomendada (4X) dos herbicidas cloransulam-methyl, chlorimuron-ethyl, imazethapyr e iodosulfuronmethyl-sodium + foramsulfuron. Londrina-PR, 2004

\begin{tabular}{|c|c|c|c|c|c|c|c|c|c|c|c|c|c|c|c|c|c|c|c|c|c|c|c|c|}
\hline \multirow{3}{*}{ Dose } & \multicolumn{6}{|c|}{ Cloransulam-methyl } & \multicolumn{6}{|c|}{ Chlorimuron-ethyl } & \multicolumn{6}{|c|}{ Imazethapyr } & \multicolumn{6}{|c|}{ Iodosulfuron + Foramsulfuron } \\
\hline & \multicolumn{2}{|c|}{7 DAT } & \multicolumn{2}{|c|}{ 14 DAT } & \multicolumn{2}{|c|}{$21 \mathrm{DAT}$} & \multicolumn{2}{|c|}{7 DAT } & \multicolumn{2}{|c|}{ 14 DAT } & \multicolumn{2}{|c|}{$21 \mathrm{DAT}$} & \multicolumn{2}{|c|}{$7 \mathrm{DAT}$} & \multicolumn{2}{|c|}{14 DAT } & \multicolumn{2}{|c|}{$21 \mathrm{DAT}$} & \multicolumn{2}{|c|}{7 DAT } & \multicolumn{2}{|c|}{ 14 DAT } & \multicolumn{2}{|c|}{$21 \mathrm{DAT}$} \\
\hline & $\mathrm{R}$ & $\mathrm{s}$ & $\mathrm{R}$ & $\mathrm{S}$ & $\mathrm{R}$ & $\mathrm{s}$ & $\mathrm{R}$ & $\mathrm{s}$ & $\mathrm{R}$ & $\mathrm{S}$ & $\mathrm{R}$ & $\mathrm{S}$ & $\mathrm{R}$ & $\mathrm{S}$ & $\mathrm{R}$ & $\mathrm{S}$ & $\mathrm{R}$ & $\mathrm{S}$ & $\mathrm{R}$ & $\mathrm{S}$ & $\mathrm{R}$ & $\mathrm{S}$ & $\mathrm{R}$ & $\mathrm{S}$ \\
\hline 0 & 0 & 0 & 0 & 0 & 0 & 0 & 0 & 0 & 0 & 0 & 0 & 0 & 0 & 0 & 0 & 0 & 0 & 0 & 0 & 0 & 0 & 0 & 0 & 0 \\
\hline 1X & 0 & 58,3 & 0 & 97,3 & 0 & 99,0 & 0 & 60,0 & 0 & 90,0 & 0 & 98,6 & 0 & 60,0 & 0 & 91,6 & 0 & 86,6 & 0 & 68,3 & 0 & 86,6 & 0 & 98,0 \\
\hline $2 \mathrm{X}$ & 0 & 66,6 & 0 & 98,6 & 0 & 100,0 & 0 & 63,3 & 0 & 93,3 & 0 & 99,3 & 0 & 61,6 & 0 & 93,3 & 0 & 90,0 & 0 & 51,6 & 0 & 90,0 & 0 & 90,0 \\
\hline $4 X$ & 0 & 61, & 0 & 99,0 & 0 & 100,0 & ") & 63,3 & ' & 96,0 & 0 & 100,0 & 0 & 60,0 & 0 & 93,3 & 0 & 96,0 & 0 & 43,3 & 0 & 96,0 & 0 & 95,0 \\
\hline
\end{tabular}

Tabela 2 - Porcentagem de controle obtida na dose recomendada dos herbicidas e diferencial de controle (S-R) aos 7, 14 e 21 dias após a aplicação dos tratamentos (DAT) nos biótipos resistente (R) e suscetível (S) da losna-branca. Londrina-PR, 2004

\begin{tabular}{|l|c|c|c|c|c|c|c|c|c|}
\hline \multirow{2}{*}{ Herbicida } & \multicolumn{3}{|c|}{7 DAT } & \multicolumn{3}{|c|}{14 DAT } & \multicolumn{3}{c|}{21 DAT } \\
\cline { 2 - 9 } & $\mathrm{R}$ & $\mathrm{S}$ & $\mathrm{S}-\mathrm{R}$ & $\mathrm{R}$ & $\mathrm{S}$ & $\mathrm{S}-\mathrm{R}$ & $\mathrm{R}$ & $\mathrm{S}$ & $\mathrm{S}-\mathrm{R}$ \\
\hline Cloransulam-methyl & 0,0 & 58,3 & 58,3 & 0,0 & 97,3 & 97,3 & 0,0 & 99,0 & 99,0 \\
\hline Chlorimuron-ethyl & 0,0 & 60,0 & 60,0 & 0,0 & 90,0 & 90,0 & 0,0 & 98,6 & 98,6 \\
\hline Imazethapyr & 0,0 & 60,0 & 60,0 & 0,0 & 91,6 & 91,6 & 0,0 & 86,6 & 86,6 \\
\hline Iodosulfuron + Foramsulfuron & 0,0 & 68,3 & 68,3 & 0,0 & 86,6 & 86,6 & 0,0 & 98,0 & 98,0 \\
\hline
\end{tabular}

Tabela 3 - Doses (g i.a. ha ${ }^{-1}$ ou g p.c. ha ${ }^{-1}$ ) correspondentes ao $\mathrm{GR}_{50}$ dos biótipos resistente (R) e suscetível (S) da losna-branca e a relação R/S. Londrina-PR, 2004

\begin{tabular}{|c|c|c|c|}
\hline Herbicida & $\mathrm{R}$ & $\mathrm{S}$ & $\mathrm{R} / \mathrm{S}$ \\
\hline Cloransulam-methyl & $>134,4$ & 7,13 & $>18,84$ \\
\hline Chlorimuron-ethyl & $>80,0$ & 3,91 & $>20,46$ \\
\hline Imazethapyr & $>400,0$ & 23,97 & $>16,68$ \\
\hline Iodosulfuron + Foramsulfuron & $>600,0$ & 30,04 & $>19,97$ \\
\hline
\end{tabular}

A dose de chlorimuron-ethyl capaz de reduzir em $50 \%$ a biomassa seca do biótipo suscetível foi de 3,9 g i.a. ha ${ }^{-1}$ e, para o biótipo com suspeita de resistência, superior à maior dose aplicada (80,0 g i.a. ha $\left.{ }^{-1}\right)$.

Para o imazethapyr e a mistura formulada de iodosulfuron-methyl-sodium mais foramsulfuron, os valores relativos ao $\mathrm{GR}_{50}$ para o biótipo suscetível foram de 23,9 e 30,0 g i.a. ha ${ }^{-1}$, respectivamente; em relação às plantas com suspeita de resistência, esses valores foram superiores a 400 e 600 g i.a. ha ${ }^{-1}$.

A relação $\mathrm{R} / \mathrm{S}$ significa quantas vezes a dose aplicada sobre o biótipo resistente deve ser superior àquela aplicada sobre o suscetivel para proporcionar o mesmo resultado. $\mathrm{O}$ herbicida com maior relação $\mathrm{R} / \mathrm{S}$ foi $\mathrm{o}$ chlorimuron-ethyl $(>20,5)$, seguido de iodosulfuron-methyl-sodium mais foramsulfuron $(>19,9)$, cloransulam-methyl $(>18,8)$ e imazethapyr $(>16,6)$ (Tabela 3). Isso significa que, para atingir $50 \%$ de redução da biomassa seca da população com suspeita de resistência, há necessidade da aplicação de doses muito maiores que as aplicadas sobre a população suscetível. Assim, pode-se dizer que o biótipo resistente apresentou diferentes níveis de resistência cruzada aos herbicidas cloransulam-methyl, chlorimuron-ethyl, imazethapyr e iodosulfuron-methyl-sodium mais foramsulfuron.

Uma das estratégias recomendadas para o controle de plantas daninhas resistentes é a combinação de herbicidas de diferentes mecanismos de ação (SBCPD, 2000). Gazziero et al. (2003) confirmaram a eficiência da estratégia proposta ao estudar alternativas químicas para o manejo de Bidens subalternans nas culturas de soja e milho. Nesse experimento, foi aplicado o herbicida 2,4-D na dose de 536,0 g e.a. ha-1. Esse herbicida, pertencente aos reguladores do crescimento, foi eficaz no controle da losna-branca. Aos 21 DAT, foram obtidas porcentagens de controle de 96 e 98\% para os biótipos considerados resistente e suscetível, respectivamente (Tabela 4). Essa 
mesma dose de 2,4-D proporcionou redução considerável da biomassa seca da parte aérea aos 30 DAT, obtendo-se valores próximos de $1 \mathrm{~g}$ por vaso para ambos os biótipos. Assim, esse herbicida pode se constituir em alternativa aos herbicidas inibidores da ALS para o controle dessa espécie em áreas com a presença de populações resistentes de losnabranca.
As curvas de dose-resposta do biótipo resistente apresentaram valores sempre inferiores aos do biótipo suscetivel, em todas as doses dos herbicidas (Figuras 1 e 2). Conclui-se que o biótipo de losna-branca estudado é resistente aos herbicidas inibidores da ALS. Os herbicidas pertencentes aos grupos químicos das imidazolinonas (imazethapyr), triazolopirimidinas (cloransulam-methyl) e sulfoniluréias

Tabela 4 - Porcentagem de controle da losna-branca resistente (R) e suscetível (S) aos 7, 14 e 21 dias após a aplicação dos tratamentos (DAT) e fitomassa seca da parte aérea (g) aos 30 DAT, em função da dose zero (0) e da dose de 536,0 g e.a. ha ${ }^{-1}$ do herbicida 2,4-D. Londrina-PR, 2004

\begin{tabular}{|c|c|c|c|c|c|c|c|c|}
\hline \multirow{3}{*}{ Dose } & \multicolumn{4}{|c|}{ Porcentagem de controle } & \multicolumn{4}{c|}{ Fitomassa da parte aérea } \\
\cline { 2 - 9 } & \multicolumn{2}{|c|}{7 DAT } & \multicolumn{2}{|c|}{14 DAT } & \multicolumn{2}{|c|}{21 DAT } & \multicolumn{2}{|c|}{30 DAT } \\
\cline { 2 - 9 } & $\mathrm{R}$ & $\mathrm{S}$ & $\mathrm{R}$ & $\mathrm{S}$ & $\mathrm{R}$ & $\mathrm{S}$ & $\mathrm{R}$ & $\mathrm{S}$ \\
\hline 0 & 0 & 0 & 0 & 0 & 0 & 0 & 12,20 & 13,07 \\
\hline 536 & 66,67 & 70,00 & 83,33 & 98,67 & 96,00 & 98,00 & 1,27 & 1,07 \\
\hline
\end{tabular}
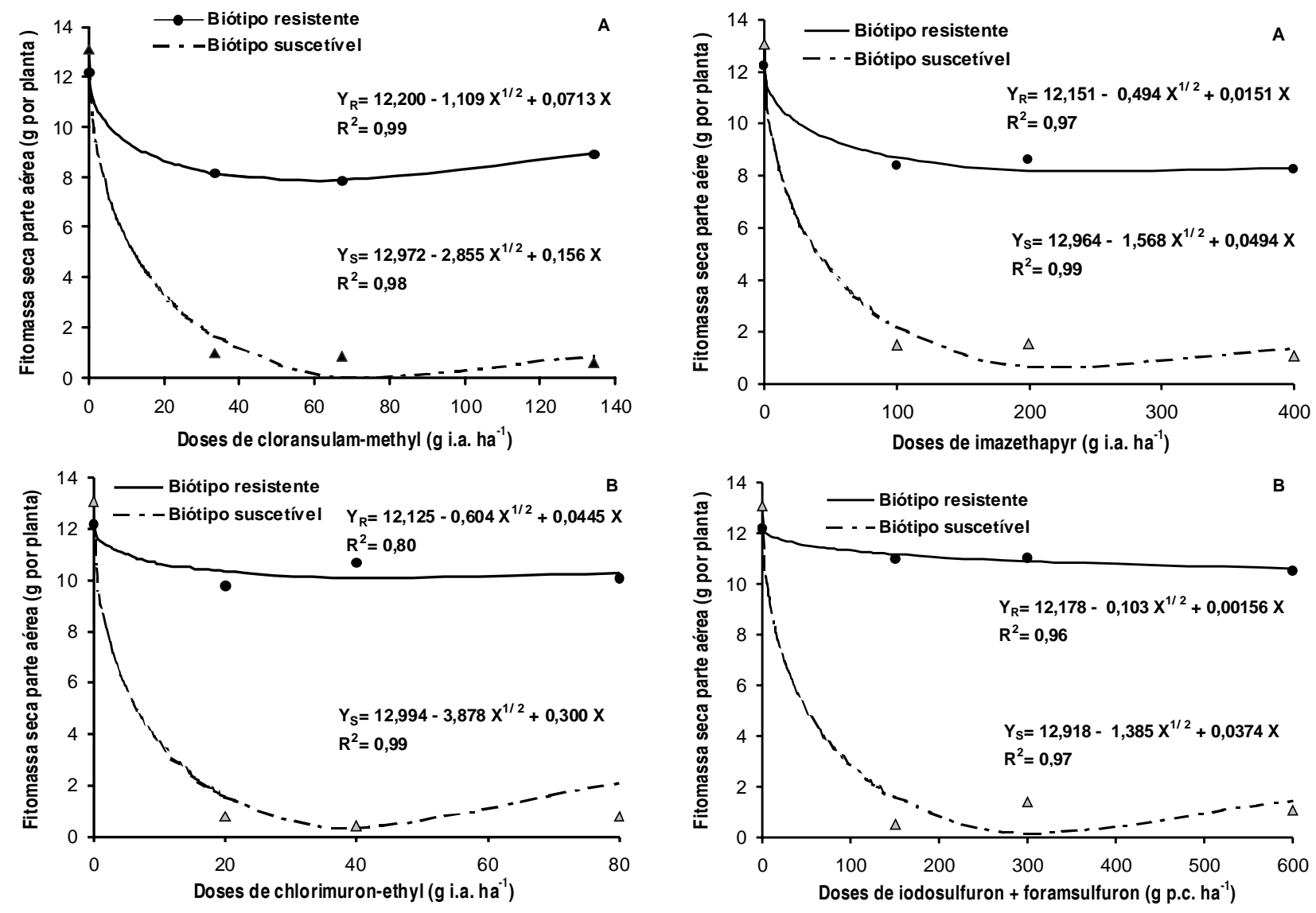

Figura 1 - Biomassa seca da parte aérea de plantas de losnabranca, em função das doses do herbicida cloransulammethyl (A) e chlorimuron-ethyl (B). Londrina-PR, 2004.

Figura 2 - Biomassa seca da parte aérea de plantas de losnabranca, em função das doses do herbicida imazethapyr (A) e iodosulfuron + foramsulfuron (B). Londrina-PR, 2004. 
(chlorimuron-ethyl e iodosulfuron-methylsodium mais foramsulfuron) apresentaram resistência cruzada aos herbicidas desses grupos químicos. Em relação ao herbicida 2,4-D, que apresenta mecanismo de ação diferente, ocorreu alto índice de controle de ambos os biótipos de losna-branca avaliados, confirmando que a alteração do mecanismo de ação do herbicida é uma importante alternativa para manejar áreas com problemas de resistência.

\section{LITERATURA CITADA}

ADKINS, S. W. et al. Weeds resistant to chlorsulfuron and atrazine from the north-east grain region of Australia. Weed Res., v. 37, p. 343-349, 1997.

BLAIR, W. Herbicide resistance in weeds: an overview. New Jersey: American Cyanamid, Agricultural Division, Crop Protection Chemicals Departament. Princeton, 1991. 16 p.

BRIGHENTI, A. M.; GAZZIERO, D. L. P.; VOLL, E. Alternativas de manejo para espécies daninhas de difícil controle: Parthenium hysterophorus e Chamaesyce hirta. In: REUNIÃO DE PESQUISA DE SOJA DA REGIÃO CENTRAL DO BRASIL, 26., 2004, Ribeirão Preto. Resumos... Londrina: 2004. p. 242-243.

BUZATTI, W. J. S.; CRISTALDO, C. J.; PODOLAN, M. J. O. Resistência de Digitaria ciliaris (capim-colchão) aos herbicidas inibidores da enzima ACCase e alternativas de controle. In: CONGRESSO BRASILEIRO DA CIÊNCIA DAS PLANTAS DANINHAS, 23., 2002, Gramado.

Resumos... Londrina: Sociedade Brasileira da Ciência das Plantas Daninhas, 2002. p. 195.

CHRISTOFFOLETI, P. J. et al. Imidazolinone resistant Bidens pilosa byotipes in the Brazilian soybeans areas. In: MEETING OF THE WEED SCIENCE SOCIETY OF AMERICA, 1996, Norfolk. Abstract... Norfolk. WSSA, 1996. p. 10.

EBERHARDT, D. S. et al. Resistência de capim-arroz (Echinochloa crusgalli) ao herbicida quinclorac. In: CONGRESSO BRASILEIRO DA CIÊNCIA DAS PLANTAS DANINHAS, 22., 2000, Foz do Iguaçu. Resumos... Londrina: SBCPD, 2000. p. 512.

GAZZIERO, D. L. P. et al. Resistência da planta daninha amendoim-bravo aos herbicidas inibidores da enzima ALS. Planta Daninha, v. 16, n. 2, p. 117-125, 1998.

GAZZIERO, D. L. P. et al. Resistência da planta daninha capim-marmelada (Brachiaria plantaginea) aos herbicidas inibidores da enzima ACCase na cultura da soja. Planta Daninha, v. 18, n. 1, p. 169-180, 2000.

GAZZIERO, D. L. P.; PRETE, C. E. C.; SUMIYA, M. Manejo de Bidens subalternans aos herbicidas inibidores da acetolactato sintase. Planta Daninha, v. 21, n. 2, p. 283-292, 2003.
GELMINI, G. A. et al. Resistência de Bidens subalternans aos herbicidas inibidores da enzima acetolactato sintase utilizados na cultura da soja. Planta Daninha, v. 20, n. 2, p. 319-325, 2002.

KISSMANN, K. G.; GROTH, D. Plantas infestantes e nocivas. 2.ed. São Paulo: BASF, 1999. T. 2. 978 p.

MAXWELL, B. D.; ROUSH, M. L.; RADOSEVICH, S. R. Predicting the evolution and dynamics of herbicide resistance in weed populations. Weed Technol., v. 4, p. 2-13, 1990.

MEROTTO Jr., A. et al. Resistência de Echinochloa sp. a quiclorac. In: CONGRESSO BRASILEIRO DA CIÊNCIA DAS PLANTAS DANINHAS, 22., 2000, Foz do Iguaçu. Resumos... Londrina: Sociedade Brasileira da Ciência das Plantas Daninhas, 2000. p. 513.

MONQUEIRO, P. A.; CHRISTOFFOLETI, P. J.; DIAS, C. T. $\mathrm{S}$. Resistência de plantas daninhas aos herbicidas inibidores da ALS na cultura da soja (Glycine max). Planta Daninha, v. 18 , n. 3, p. 419-425, 2000.

NJOROGE, J. M. Tolerance of Bidens pilosa L. and Parthenium hysterophorus L. to paraquat (Gramoxone) in Kenya coffee. Kenya Coffee, v. 56, p. 999-1001, 1991.

NOLDIN, J. A. et al. Sagitária resistente a herbicidas inibidores da ALS. In: CONGRESSO BRASILEIRO DA CIÊNCIA DAS PLANTAS DANINHAS, 22., 2000, Foz do Iguaçu. Resumos... Londrina: Sociedade Brasileira da Ciência das Plantas Daninhas, 2000. p. 514.

NOLDIN, J. A.; EBERHARDT, D. S.; RAMPELOTTI, F. T. Cyperus difformis (L.) resistente a herbicidas inibidores da ALS em Santa Catarina. In: CONGRESSO BRASILEIRO DA CIÊNCIA DAS PLANTAS DANINHAS, 23., 2002, Gramado. Resumos... Londrina: Sociedade Brasileira da Ciência das Plantas Daninhas, 2002a. p. 198.

NOLDIN, J. A.; EBERHARDT, D. S.; RAMPELOTTI, F. T. Fimbristylis miliacea (L.) Vahl resistente a herbicidas inibidores da ALS em Santa Catarina. In: CONGRESSO BRASILEIRO DA CIÊNCIA DAS PLANTAS DANINHAS, 23., 2002, Gramado. Resumos... Londrina: Sociedade Brasileira da Ciência das Plantas Daninhas, 2002b. p. 199.

ROMAN, E. S. et al. Resistência de azevém (Lolium multiflorum) ao herbicida glyphosate. Planta Daninha, v. 22, n. 2, p. 301-306, 2004.

SOCIEDADE BRASILEIRA DA CIÊNCIA DA PLANTA DANINHA - SBCPD. Identificação e manejo de plantas daninhas resistentes aos herbicidas. Viçosa, MG: 2000. $32 \mathrm{p}$.

VARGAS, L. et al. Identificação de biótipos de azevém (Lolium multiflorum) resistentes ao herbicida glyphosate em pomares de maçã. Planta Daninha, v. 22, n. 4, p. 617-622, 2004.

VIDAL, R. A.; MEROTO JR, A. Resistência de amendoimbravo aos herbicidas inibidores da enzima acetolactato sintase. Planta Daninha, v. 17, n. 3, p. 367-373, 1999. 